\title{
The IDC Seismic, Hydroacoustic and Infrasound Global Low and High Noise Models
}

\author{
David Brown, ${ }^{1}$ Lars Ceranna, ${ }^{2}$ Mark Prior, ${ }^{1}$ Pierrick Mialle, ${ }^{1}$ and Ronan J. Le Bras ${ }^{1}$
}

\begin{abstract}
The International Data Centre (IDC) in Vienna, Austria, is determining, as part of automatic processing, sensor noise levels for all seismic, hydroacoustic, and infrasound (SHI) stations in the International Monitoring System (IMS) operated by the Provisional Technical Secretariat of the Comprehensive Nuclear-Test-Ban Treaty Organization (CTBTO). Sensor noise is being determined several times per day as a power spectral density (PSD) using the Welch overlapping method. Based on accumulated PSD statistics a probability density function (PDF) is also determined, from which low and high noise curves for each sensor are extracted. Global low and high noise curves as a function of frequency for each of the SHI technologies are determined as the minimum and maximum of the individual station low and high noise curves, respectively, taken over the entire network of contributing stations. An attempt is made to ensure that only correctly calibrated station data contributes to the global noise models by additionally considering various automatic detection statistics. In this paper global low and high noise curves for 2010 are presented for each of the SHI monitoring technologies. Except for a very slight deviation at the microseism peak, the seismic global low noise model returns identically the PeTERSON (1993) NLNM low noise curve. The global infrasonic low noise model is found to agree with that of Bowman et al. $(2005,2007)$ but disagrees with the revised results presented in Bowman et al. (2009) by a factor of 2 in the calculation of the PSD. The global hydroacoustic low and high noise curves are found to be in quantitative agreement with Urick's oceanic ambient noise curves for light to heavy shipping. Whale noise is found to be a feature of the hydroacoustic high noise curves at around 15 and $25 \mathrm{~Hz}$.
\end{abstract}

Key words: Seismic, infrasound, hydroacoustic, global noise models.

1 International Data Centre, Comprehensive Nuclear-TestBan Treaty Organization, Preparatory Commission, IDC/CTBTO Vienna International Centre, PO Box 1200, 1400 Vienna, Austria. E-mail: David.Brown@ctbto.org

2 Bundesanstalt für Geowissenschaften und Rohstoffe (BGR), Geozentrum Hannover Stilleweg 2, 30655 Hannover, Germany.

\section{Introduction}

The Provisional Technical Secretariat for the Preparatory Commission for the Comprehensive Nuclear-Test-Ban Treaty Organization is tasked with establishing the verification regime for the Comprehensive Nuclear-Test-Ban Treaty (CTBT) that, upon Entry Into Force (EIF), bans the detonation of nuclear devices in any environment. The framework of the verification regime is the global network of 337 seismic, hydroacoustic, infrasound, and radionuclide stations that form the International Monitoring System. The International Data Centre processes in near real time data received from the IMS stations, subsequently generating several event bulletins for the benefit of the States Parties that are signatories to the Treaty.

Along with its regular event processing, the IDC is also mandated to record and monitor station ambient noise with the expectation that knowledge of this sort may be indicative of station state of health (SOH).

Station ambient noise conditions are being represented by the power spectral density (PSD), which provides a measure of the power contained in the signal at each frequency.

Determining both single station and network low and high noise models becomes a straightforward procedure when station ambient noise information is routinely accessible. The purpose of this paper is to present the inferred global low and high noise models for each of the SHI technologies based on data recorded by the IMS network. In doing so, care is taken at all stages to ensure both the integrity of the data and the method used to determine the station noise information.

Section 2 of this paper discusses the method used in determining the station ambient noise, as well as 
tests performed to ensure that the chosen PSD method is providing the correct estimation of the PSD.

Section 3 discusses the data used during the analysis, and section four the results for each of the SHI technologies.

\section{Numerical Method and Testing}

\subsection{Numerical Method}

The Welch overlapping method (WeLCH 1967) forms the basis of the procedure used in this paper to determine the PSD. In this method the time interval spanning the data under consideration is divided into a sequence of overlapping sub-intervals and the PSD for each sub-interval is determined. The average of the sub-interval PSD's is assumed to provide an estimate of the required PSD. This averaging procedure reduces the variance on the estimated PSD, which may otherwise be of the order of the contributing sample values (WeLCH 1967). Strictly speaking, the use of the PSD requires that the waveform under consideration be a Wide-Sense Stationary Process (see, e.g., SchreIER and Scharf 2010), implying that both the mean and autocorrelation of the sampled waveform are time independent, in which case the PSD is the Fourier transform of the autocorrelation function as asserted by the Wiener-Khinchin theorem. Here we are assuming that stationarity is assumed to hold as the propagating signals are considered to be short-lived transitory phenomenon and will thus provide an only minor impact on the statistics.

When evaluating the PSD's, careful attention has been given to the spectral windowing process, which is discussed more fully in the Appendix. In this analysis we have chosen to use the nutall4a window of Heinzel et al. (2002), which is a good general purpose spectral window with spectral leakage properties superior to that of the Hanning or Hamming windows.

The PSD estimate used in this paper is given by the expression $P_{\mathrm{SD}}\left(\omega_{j}\right)=\frac{2\left|F\left(\omega_{j}\right)\right|^{2}}{\Delta I_{W}}$ where $\omega_{j}=\frac{2 \pi j}{n \Delta}$ for $j=0, \ldots, n / 2$ is the jth frequency picket, $n$ is the number of samples, $\Delta$ is the sample rate, $F$ is the output of a unitary Fourier Transform algorithm and
$I_{\mathrm{w}}$ the sum of the squares of the spectral window coefficients. This expression is discussed further in the Appendix and derived more fully in references like HARRIs (1978), and HeINZEl et al. (2002).

\subsection{Testing}

Two levels of testing have been applied to the algorithm described in Sect. 2.1. The first is against an artificial dataset intended to mimic digitizer noise for which the PSD has a theoretically determinable expression. The second is a blind test with a second algorithm on an otherwise random selected data set.

\subsubsection{Test 1: Digitizer Noise}

The procedure described in HeInzel et al. (2002) has been used to test the algorithm. Here, each sample in a synthetic time-series data set has been rounded to multiples of a parameter $U_{0}$ defined a priori, which represents the least-significant-bit of a digitizer. In this case, the PSD has a noise-floor that is given by the expression (see, e.g., Lyons 1997) $U^{2}(\omega)=\frac{U_{0}^{2}}{6 \Delta}$, where $\omega$ is angular frequency.

The following strategy outlined in HeInzel et al., (2002) was used to generate the synthetic time-series data set:

1. The double-sinusoid $u(t)=A_{1} \sin \left(2 \pi f_{1} t\right)+A_{2} \sin$ $\left(2 \pi f_{2} t\right)$ is used to provide the basic analogue signal. Here, $f_{1}=0.3123456 \mathrm{~Hz}, f_{2}=2.0 \mathrm{~Hz}$, $A_{1}=2.123456, A_{2}=1.0$ is used.

2. $u(t)$ has been sampled at $20 \mathrm{~Hz}$ to provide the time-series: $u_{j}=A_{1} \sin \left(\frac{2 \pi f_{1} j}{\Delta}\right)+A_{2} \sin \left(\frac{2 \pi f_{2} j}{\Delta}\right)$ for $j=1, \ldots, N$.

3. The new time-series $y_{j}$ for $j=1, \ldots, N$ is formed, where $y_{j}=\operatorname{int}\left[\frac{u_{j}}{U_{0}}+0.5\right] U_{0}$.

4. With the values for $A_{1}, A_{2}, f_{1}, f_{2}, U_{0}$, and $\Delta$ as given above we should expect a white noise background with $\log _{10}$ PSD $=\log _{10}\left(\frac{U_{0}}{6 \Delta}\right)=-8.079$.

With this procedure a time-series with duration 1-h was generated. After passing the algorithm as described above over the synthetic data set, the PSD as shown in Fig. 1 was obtained.

The desired noise floor is accurately rendered, and further, when multiplying by the Equivalent Noise Bandwidth for the nutall4a window (see the 


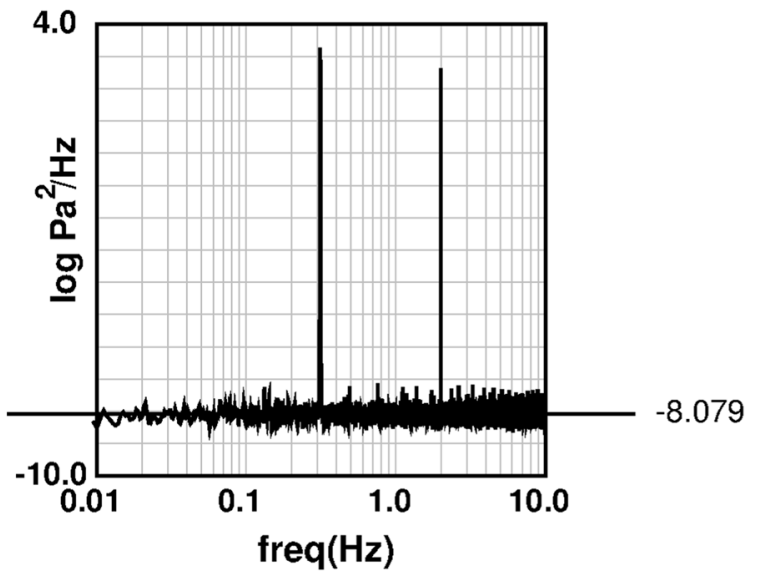

Figure 1
Power spectral density as a function of frequency for the Test 1
data set

Appendix), the amplitude of the spectral peaks is accurately determined, as is shown in Fig. 1.

\subsubsection{Test 2: Blind Test with a Second Independent PSD Algorithm}

One-hour data segments were chosen randomly from the list of infrasound stations provided in Table 1. A completely independent second algorithm that uses the matlab pwelch function (MATLAB 2008) to estimate PSD's (denoted as BGR in what follows) was provided by one of the authors and compared with the algorithm described above (denoted CTBTO in what follows). The results are shown in Fig. 2 and indicate excellent agreement.

We conclude from these two tests that the algorithm is functioning, as it should.

\section{The Data}

The IMS seismic, hydroacoustic and infrasound stations that were used in the current analysis are listed in Table 1; the locations of the stations is shown in Fig. 3.

Both primary and auxiliary seismic stations were used in this analysis, and although data from the primary stations is continuously recorded, the auxiliary stations generally send data upon request in order to refine knowledge of a seismic event during the analysis stage. Data from the auxiliary seismic stations may therefore not be present for the entire analysis period.

Data sampled four times per day for the whole of 2010 were used in the current analysis except in situations where better resolution of the low or high noise models was required for a particular station where the data was sampled each hour for the entire year.

Each sampling consisted of 1-h of waveform data divided into 3-min overlapping segments as outlined above, with the seismic data being deconvolved to acceleration and the infrasound and hydroacoustic data deconvolved to displacement, the instrument response has been removed in each case. Note that in the case of the infrasound data only the response of the sensor and digitizer has been removed, not that of the spatial filter system that was likely to be present.

\section{Low and High Noise Models}

Probability Density Functions using the procedure discussed by McNAMARA and Buland (2004) are determined for each SHI sensor. Data displayed in this format allows a ready estimate of the sensor low and high noise models for the period in which data was contributed. As an example, Fig. 4 shows the PDF obtained for sensor I02AR/I02H1 for the year 2010. The low and high noise curves as a function of frequency are determined by plotting the PDF function at the 5 and $95 \%$ probability levels, respectively. The global low noise curve as a function of frequency is defined to be, for a given frequency, the minimum of the low noise curves from all contributing sensors across all stations at the given frequency. Only stations with sufficient contributing data such that a well-defined PDF is formed with well-behaved low and high noise curves were used in the analysis.

\subsection{Seismic}

A random shift of up to $6 \mathrm{~h}$ is applied to the requested processing time for the seismic data in order to reduce the likelihood of contamination by regular cultural noise. Only vertical channels were considered when performing the seismic analysis, 
Table 1

IMS seismic, hydroacoustic and infrasound stations that were used in the current analysis

\begin{tabular}{|c|c|c|c|c|c|}
\hline Station & Location & State & Latitude & Longitude & Station type \\
\hline AKASG & Malin & Ukraine & 50.7012 & 29.2242 & Primary seismic \\
\hline ARCES & Finnmark & Norway & 69.5348 & 25.5057 & Primary seismic \\
\hline ASAR & Alice Springs & Australia & -23.665134 & 133.90526 & Primary seismic \\
\hline BDFB & Brasilia & Brazil & -15.64178 & -48.01485 & Primary seismic \\
\hline BOSA & Boshof & South Africa & -28.61405 & 25.25542 & Primary seismic \\
\hline BRTR & Belbashi & Turkey & 39.725 & 33.639 & Primary seismic \\
\hline CMAR & Chiang Mai & Thailand & 18.4576 & 98.94315 & Primary seismic \\
\hline CPUP & Villa Florida & Paraguay & -26.3307 & -57.331 & Primary seismic \\
\hline DBIC & Dimbroko & Cote d'Ivoire & 6.6701 & -4.8563 & Primary seismic \\
\hline ESDC & Sonseca & Spain & 39.6744 & -3.963 & Primary seismic \\
\hline FINES & Lahti & Finland & 61.4436 & 26.0771 & Primary seismic \\
\hline GERES & Freyung & Germany & 48.845106 & 13.701559 & Primary seismic \\
\hline GEYT & Alibeck & Turkmenistan & 37.92955 & 58.11706 & Primary seismic \\
\hline ILAR & Eielson & United States of America & 64.771446 & -146.88665 & Primary seismic \\
\hline KBZ & Khabaz & Russian Federation & 43.726898 & 42.8996 & Primary seismic \\
\hline KEST & Kesra & Tunisia & 35.7317 & 9.346 & Primary seismic \\
\hline КМВО & Kilimambogo & Kenya & -1.1268 & 37.2523 & Primary seismic \\
\hline KSRS & Wonju & Republic of Korea & 37.4421 & 127.8844 & Primary seismic \\
\hline LPAZ & $\mathrm{La} \mathrm{Paz}$ & Bolivia & -16.287927 & -68.130706 & Primary seismic \\
\hline MAW & Mawson Antarctica & Australia & -67.6046 & 62.8713 & Primary seismic \\
\hline MJAR & Matushiro & Japan & 36.524717 & 138.24718 & Primary seismic \\
\hline MKAR & Makanchi & Kazakhstan & 46.7937 & 82.2904 & Primary seismic \\
\hline NOA & Hamar & Norway & 61.0397 & 11.2148 & Primary seismic \\
\hline NVAR & Mina Nevada & United States of America & 38.429609 & -118.30355 & Primary seismic \\
\hline PDAR & Pinedale Wyoming & United States of America & 42.7667 & -109.5579 & Primary seismic \\
\hline PETK & Petropavlovsky-Kamchatskiy & Russian Federation & 53.108215 & 157.69885 & Primary seismic \\
\hline PLCA & Paso Flores & Argentina & -40.732733 & -70.550835 & Primary seismic \\
\hline PPT & Tahiti & France & -17.5896 & -149.5764 & Primary seismic \\
\hline ROSC & El Rosal & Columbia & 4.844856 & -74.321203 & Primary seismic \\
\hline SCHQ & Schefferville Quebec & Canada & 54.832402 & -66.833177 & Primary seismic \\
\hline SONM & Songino & Mongolia & 47.83469 & 106.39499 & Primary seismic \\
\hline STKA & Stephens Creek & Australia & -31.8743 & 141.5964 & Primary seismic \\
\hline TORD & Torodi & Niger & 13.14771 & 1.6947087 & Primary seismic \\
\hline TXAR & Lajitas Texas & United States of America & 29.333965 & -103.66769 & Primary seismic \\
\hline ULM & Lac Du Bonnet, Manitoba & Canada & 50.250261 & -95.874956 & Primary seismic \\
\hline USRK & Ussuriysk & Russian Federation & 44.1998 & 131.9888 & Primary seismic \\
\hline VNDA & Vanda, Antarctica & United States of America & -77.5173 & 161.8528 & Primary seismic \\
\hline WRA & Warramunga NT & Australia & -19.942589 & 134.33951 & Primary seismic \\
\hline YKA & Yellowknife & Canada & 62.4931 & -114.6062 & Primary seismic \\
\hline ZALV & Zalesovo & Russian Federation & 53.948063 & 84.818807 & Primary seismic \\
\hline AAK & Ala-Archa & Kyrgyzstan & 42.6391 & 74.4942 & Auxiliary seismic \\
\hline AFI & Afiamalu & Samoa & -13.9093 & -171.7773 & Auxiliary seismic \\
\hline AKTO & Aktyubinsk & Kazakhstan & 50.4348 & 58.0164 & Auxiliary seismic \\
\hline ANMO & Albuquerque, New Mexico & United States of America & 34.9462 & -106.4567 & Auxiliary seismic \\
\hline ASF & Tel Al Asfar & Jordan & 32.1723 & 36.8972 & Auxiliary seismic \\
\hline ATAH & Atahualpa & Peru & -7.13506 & -78.39445 & Auxiliary seismic \\
\hline ATD & Arta Tunnel & Djibouti & 11.53 & 42.847 & Auxiliary seismic \\
\hline BATI & Baumata, Nusa Tengarra & Indonesia & -10.206 & 123.6627 & Auxiliary seismic \\
\hline BBB & Bella Bella & Canada & 52.1847 & -128.1133 & Auxiliary seismic \\
\hline BBTS & Babate & Senegal & 14.6604 & -16.5334 & Auxiliary seismic \\
\hline BORG & Borganes & Iceland & 64.7474 & -21.3268 & Auxiliary seismic \\
\hline BVAR & Borovoye & Kazakhstan & 53.0249 & 70.3885 & Auxiliary seismic \\
\hline CFAA & Coronel Fontana & Argentina & -31.60475 & -68.23756 & Auxiliary seismic \\
\hline CMIG & Colonia Cuauhtemoc, Oaxaca & Mexico & 17.091 & -94.8838 & Auxiliary seismic \\
\hline CTA & Charters Towers & Australia & -20.0876 & 146.25 & Auxiliary seismic \\
\hline
\end{tabular}


Table 1 continued

\begin{tabular}{|c|c|c|c|c|c|}
\hline Station & Location & State & Latitude & Longitude & Station type \\
\hline DAVOX & Davos & Switzerland & 46.7806 & 9.8797 & Auxiliary seismic \\
\hline DLBC & Dease Lake, British Columbia & Canada & 58.43696 & -130.03051 & Auxiliary seismic \\
\hline DZM & Mont Dzumad & New Caledonia & -22.068 & 166.4469 & Auxiliary seismic \\
\hline EIL & Eilath & Israel & 29.6725 & 34.9519 & Auxiliary seismic \\
\hline EKA & Eskdalemuir & United Kingdom & 55.3332 & -3.1588 & Auxiliary seismic \\
\hline ELK & Elko, Nevada & United States of America & 40.7448 & -115.2388 & Auxiliary seismic \\
\hline FITZ & Fitzroy Crossing & Australia & -18.09826 & 125.6403 & Auxiliary seismic \\
\hline FRB & Iqaluit & Canada & 63.7467 & -68.5467 & Auxiliary seismic \\
\hline GNI & Garni & Armenia & 40.1495 & 44.7414 & Auxiliary seismic \\
\hline GUMO & Guam, Marianas Islands & United States of America & 13.5892 & 144.8684 & Auxiliary seismic \\
\hline HFS & Hagfors & Sweden & 60.133474 & 13.69449 & Auxiliary seismic \\
\hline HNR & Honiara & Solomon Islands & -9.4322 & 159.9471 & Auxiliary seismic \\
\hline IDI & Anóyia & Greece & 35.288 & 24.89 & Auxiliary seismic \\
\hline INK & Inuvik, Northwest Territory & Canada & 68.306516 & -133.52543 & Auxiliary seismic \\
\hline JCJ & Chichijima, Ogasawara & Japan & 27.095467 & 142.18463 & Auxiliary seismic \\
\hline JKA & Kamikawa-Asahi, Hokkaido & Japan & 44.11895 & 142.59325 & Auxiliary seismic \\
\hline JMIC & Jan Mayen & Norway & 70.9866 & -8.50515 & Auxiliary seismic \\
\hline JNU & Ohita, Kyushu & Japan & 33.121667 & 130.87833 & Auxiliary seismic \\
\hline JOW & Kunigami, Okinawa & Japan & 26.836 & 128.2731 & Auxiliary seismic \\
\hline JTS & Las Juntas de Abangares & Costa Rica & 10.2908 & -84.9525 & Auxiliary seismic \\
\hline KAPI & Kappang, Sulawesi & Indonesia & -5.0142 & 119.7517 & Auxiliary seismic \\
\hline KDAK & Kodiak Island, Alaska & United States of America & 57.7828 & -152.5835 & Auxiliary seismic \\
\hline KURK & Kurchatov & Kazakhstan & 50.62264 & 78.53039 & Auxiliary seismic \\
\hline KVAR & Kislovodsk, Stavropol'skiy & Russian Federation & 43.9557 & 42.6952 & Auxiliary seismic \\
\hline LBTB & Lobatse & Botswana & -25.0151 & 25.5966 & Auxiliary seismic \\
\hline LEM & Lembang, Jawa Barat & Indonesia & -6.82645 & 107.61748 & Auxiliary seismic \\
\hline LPIG & La Paz, Baja California Sur & Mexico & 24.10103 & -110.30931 & Auxiliary seismic \\
\hline LSZ & Lusaka & Zambia & -15.2766 & 28.1882 & Auxiliary seismic \\
\hline MATP & Matopo & Zimbabwe & -20.42583 & 28.49944 & Auxiliary seismic \\
\hline MBAR & Mbarara & Uganda & -0.6019 & 30.7382 & Auxiliary seismic \\
\hline MDT & Midelt & Morocco & 32.814 & -4.607 & Auxiliary seismic \\
\hline MLR & Muntele Rosu & Romania & 45.4917 & 25.9437 & Auxiliary seismic \\
\hline MMAI & Mount Meron & Israel & 33.01518 & 35.40311 & Auxiliary seismic \\
\hline MSKU & MasUnited Kingdomu & Gabon & -1.6557 & 13.6116 & Auxiliary seismic \\
\hline NEW & Newport, Washington & United States of America & 48.26333 & -117.12 & Auxiliary seismic \\
\hline NNA & Nana & Peru & -11.9873 & -76.8422 & Auxiliary seismic \\
\hline NWAO & Narrogin & Australia & -32.9277 & 117.239 & Auxiliary seismic \\
\hline OBN & Obninsk & Russian Federation & 55.1138 & 36.5687 & Auxiliary seismic \\
\hline OPO & Ambohidratompo & Madagascar & -18.5706 & 47.1879 & Auxiliary seismic \\
\hline PALK & Pallekele & Sri Lanka & 7.2728 & 80.7022 & Auxiliary seismic \\
\hline PCRV & Puerto la Cruz & Venezuela & 10.1634 & -64.58963 & Auxiliary seismic \\
\hline PFO & Pinon Flat, California & United States of America & 33.6092 & -116.4553 & Auxiliary seismic \\
\hline PMG & Port Moresby & Papua New Guinea & -9.4092 & 147.1539 & Auxiliary seismic \\
\hline PMSA & Palmer Station, Antarctica & United States of America & -64.7742 & -64.049 & Auxiliary seismic \\
\hline PSI & Parapat, Sumatra & Indonesia & 2.6952 & 98.924 & Auxiliary seismic \\
\hline QSPA & South Pole, Antarctica & United States of America & -89.9279 & 145.0 & Auxiliary seismic \\
\hline RAO & Raoul, Kermadec Islands & New Zealand & -29.2517 & -177.9183 & Auxiliary seismic \\
\hline RAR & Raratonga & Cook Islands & -21.2125 & -159.7733 & Auxiliary seismic \\
\hline RCBR & Riachuelo & Brazil & -5.82739 & -35.90131 & Auxiliary seismic \\
\hline RES & Resolute, Nunavut & Canada & 74.689233 & -94.896167 & Auxiliary seismic \\
\hline RPN & Easter Island & Chile & -27.1267 & -109.3344 & Auxiliary seismic \\
\hline RPZ & Rata Peaks & New Zealand & -43.7146 & 171.054 & Auxiliary seismic \\
\hline SADO & Sadowa & Canada & 44.7694 & -79.1417 & Auxiliary seismic \\
\hline SEY & Seymchan & Russian Federation & 62.9328 & 152.3822 & Auxiliary seismic \\
\hline SFJD & Søndre Strømford & Greenland & 66.995999 & -50.6215 & Auxiliary seismic \\
\hline SIV & San Ignacio & Bolivia & -15.991 & -61.072 & Auxiliary seismic \\
\hline SJG & San Juan & Puerto Rico & 18.1117 & -66.15 & Auxiliary seismic \\
\hline
\end{tabular}


Table 1 continued

\begin{tabular}{|c|c|c|c|c|c|}
\hline Station & Location & State & Latitude & Longitude & Station type \\
\hline SNAA & Sanae Station, Antarctica & Germany/South Africa & -71.6707 & -2.8379 & Auxiliary seismic \\
\hline SPITS & Spitsbergen & Norway & 78.1777 & 16.37 & Auxiliary seismic \\
\hline SUR & Sutherland & South Africa & -32.3797 & 20.8117 & Auxiliary seismic \\
\hline TEIG & Tepich, Yucatan & Mexico & 20.2264 & -88.2776 & Auxiliary seismic \\
\hline TGY & Tagatay City & Philippines & 14.1008 & 120.93837 & Auxiliary seismic \\
\hline TKL & Tuckaleechee Caverns, Tennessee & United States of America & 35.658 & -83.774 & Auxiliary seismic \\
\hline TSUM & Tsumeb & Namibia & -19.2022 & 17.5838 & Auxiliary seismic \\
\hline URZ & Urewera & New Zealand & -38.2592 & 177.1109 & Auxiliary seismic \\
\hline USHA & Ushuaia & Argentina & -54.83192 & -68.43432 & Auxiliary seismic \\
\hline VAE & Valguarnera & Italy & 37.469 & 14.3533 & Auxiliary seismic \\
\hline VRAC & Vranov & Czech Republic & 49.30828 & 16.59351 & Auxiliary seismic \\
\hline YBH & Yreka Blue Horn & United States of America & 41.73193 & -122.71038 & Auxiliary seismic \\
\hline I02AR & Ushuaia & Argentina & -54.58057 & -67.30923 & Infrasound \\
\hline I04AU & Shannon & Australia & -34.59761 & 116.35669 & Infrasound \\
\hline I05AU & Hobart & Australia & -42.490798 & 147.68063 & Infrasound \\
\hline I07AU & Warramunga NT & Australia & -19.93482 & 134.32953 & Infrasound \\
\hline I08BO & Penas-Bolivia & Bolivia & -16.21523 & -68.45345 & Infrasound \\
\hline I09BR & Brasilia & Brazil & -15.637967 & -48.016422 & Infrasound \\
\hline I10CA & Lac Du Bonnet & Canada & 50.201469 & -96.026854 & Infrasound \\
\hline I11CV & Maio & Cape Verde & 15.25729 & -23.18388 & Infrasound \\
\hline I13CL & Easter Island & Chile & -27.12726 & -109.36265 & Infrasound \\
\hline I14CL & Robinson Carusoe Island & Chile & -33.65379 & -78.79598 & Infrasound \\
\hline I17CI & Dimbokro & Ivory Coast & 6.6703566 & -4.8569106 & Infrasound \\
\hline I18DK & Qaanaaq & Greenland & 77.47556 & -69.28776 & Infrasound \\
\hline I21FR & Marquesas Islands & France & -8.86783 & -140.15907 & Infrasound \\
\hline I22FR & Port Laguerre New Caldeonoia & FRANCE & -22.18445 & 166.84592 & Infrasound \\
\hline I23FR & Kerguelen & France & -49.34578 & 70.24159 & Infrasound \\
\hline I24FR & Tahiti & France & -17.74929 & -149.29582 & Infrasound \\
\hline $\mathrm{I} 26 \mathrm{DE}$ & Freyung & Germany & 48.851617 & 13.713128 & Infrasound \\
\hline I27DE & Georg von Neumayer Antarctica & Germany & -70.7011 & -8.30291 & Infrasound \\
\hline I30JP & Isumi & Japan & 35.307756 & 140.31376 & Infrasound \\
\hline $\mathrm{I} 31 \mathrm{KZ}$ & Aktyubinsk & Kazakhstan & 50.40697 & 58.03482 & Infrasound \\
\hline I32KE & Nairobi & Kenya & -1.24216 & 36.82721 & Infrasound \\
\hline I33MG & Antananarivo & Madagascar & -19.010859 & 47.305024 & Infrasound \\
\hline I34MN & Songino & Mongolia & 47.80172 & 106.41012 & Infrasound \\
\hline I35NA & Tsumeb & Namibia & -19.19135 & 17.57678 & Infrasound \\
\hline $\mathrm{I} 36 \mathrm{NZ}$ & Chatham Islands & New Zealand & -43.91662 & -176.48337 & Infrasound \\
\hline I39PW & Palau & Palau & 7.53547 & 134.54704 & Infrasound \\
\hline I41PY & Villa Florida & Paraguay & -26.3423 & -57.31188 & Infrasound \\
\hline I43RU & Dubna & Russian Federation & 56.72136 & 37.21759 & Infrasound \\
\hline I44RU & Petropavlovsk-Kamchatsky & Russian Federation & 53.1058 & 157.7139 & Infrasound \\
\hline I45RU & Ussuriysk & Russian Federation & 44.1999 & 131.9773 & Infrasound \\
\hline I46RU & Zalesovo & Russian Federation & 53.94872 & 84.81891 & Infrasound \\
\hline I47ZA & Boshof & South Africa & -28.621123 & 25.235228 & Infrasound \\
\hline I48TN & Kesra & Tunisia & 35.80523 & 9.32302 & Infrasound \\
\hline I49 GB & Tristan Da Cunha & United Kingdom & -37.08995 & -12.33192 & Infrasound \\
\hline I50 GB & Ascension Island & United Kingdom & -7.93774 & -14.37517 & Infrasound \\
\hline I51 GB & Bermuda & United Kingdom & 32.36154 & -64.69874 & Infrasound \\
\hline I52 GB & Diego Garcia & United Kingdom & -7.37781 & 72.484161 & Infrasound \\
\hline I53US & Fairbanks Alaska & United States of America & 64.875 & -147.86114 & Infrasound \\
\hline I55US & Windless Bight Antarctica & United States of America & -77.73149 & 167.58742 & Infrasound \\
\hline I56US & Newport Washington & United States of America & 48.26408 & -117.12567 & Infrasound \\
\hline I57US & Pinon Flat California & United States of America & 33.605852 & -116.45328 & Infrasound \\
\hline I59US & Hawaii & United States of America & 19.591532 & -155.8936 & Infrasound \\
\hline H01 & Cape Leeuwin & Australia & -34.88316 & 114.13608 & Hydroacoustic \\
\hline H03 & Juan Fernandez Island & Chile & -33.825843 & -78.909483 & Hydroacoustic \\
\hline H08 & Diego Garcia & United Kingdom & -7.6275 & 72.48383 & Hydroacoustic \\
\hline
\end{tabular}


Table 1 continued

\begin{tabular}{lllccc}
\hline Station & Location & State & Latitude & Longitude & Station type \\
\hline H10 & Ascension Island & United Kingdom & -8.95274 & -14.6629 & Hydroacoustic \\
H11 & Wake Island & United States of America & 18.49568 & 166.68646 & Hydroacoustic \\
\hline
\end{tabular}
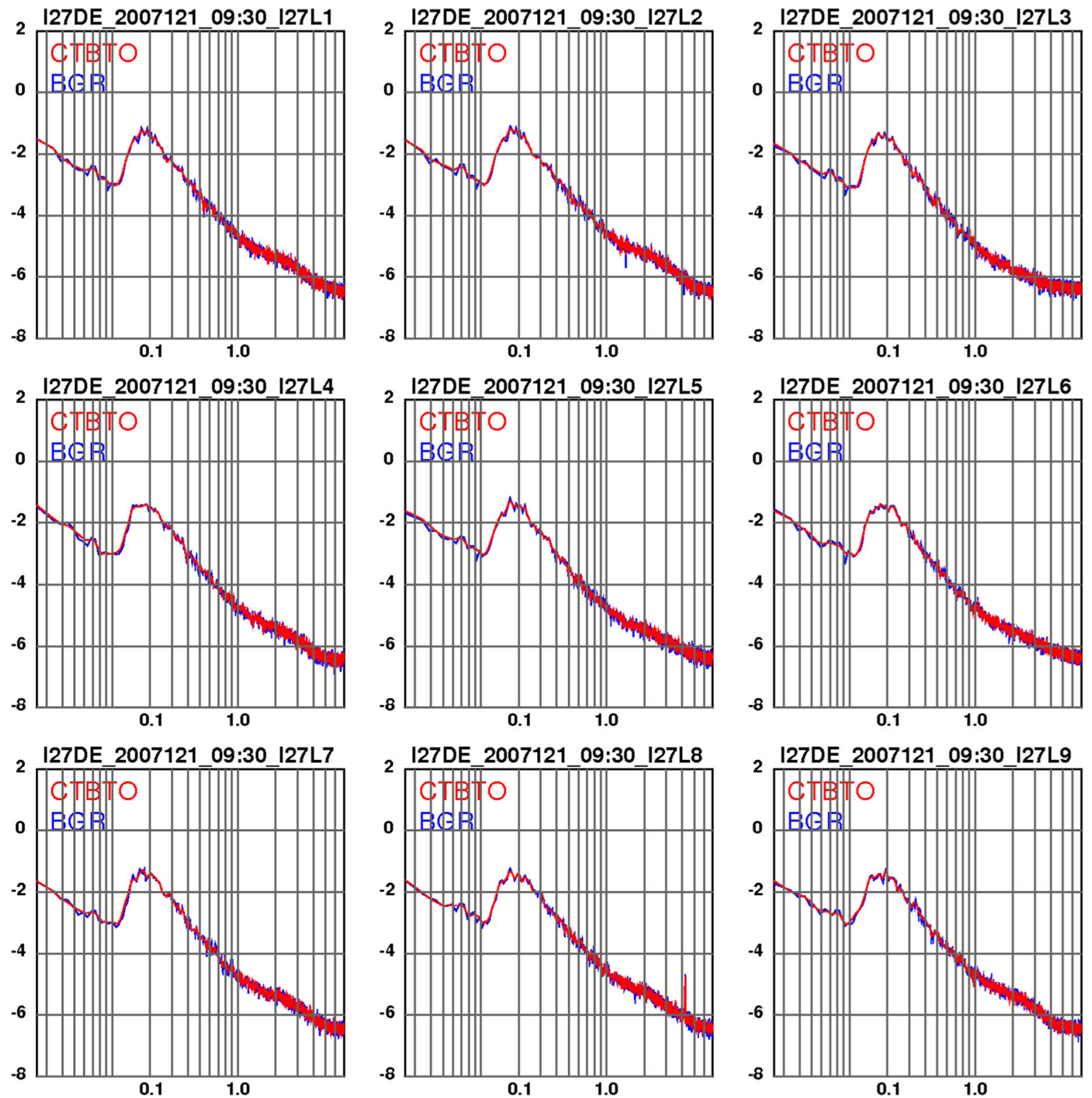

Figure 2

Results of a blind-comparison test between two different PSD algorithms on randomly chosen infrasound data. Abscissa values are frequency in $\mathrm{Hz}$, and ordinate values are logarithm base 10 of the PSD in $\mathrm{Pa}^{2}$ per $\mathrm{Hz}$. The CTBTO data refer to results generated by the procedure discussed in this Paper. The BGR data refers to a second independent algorithm provided by one of the present authors 

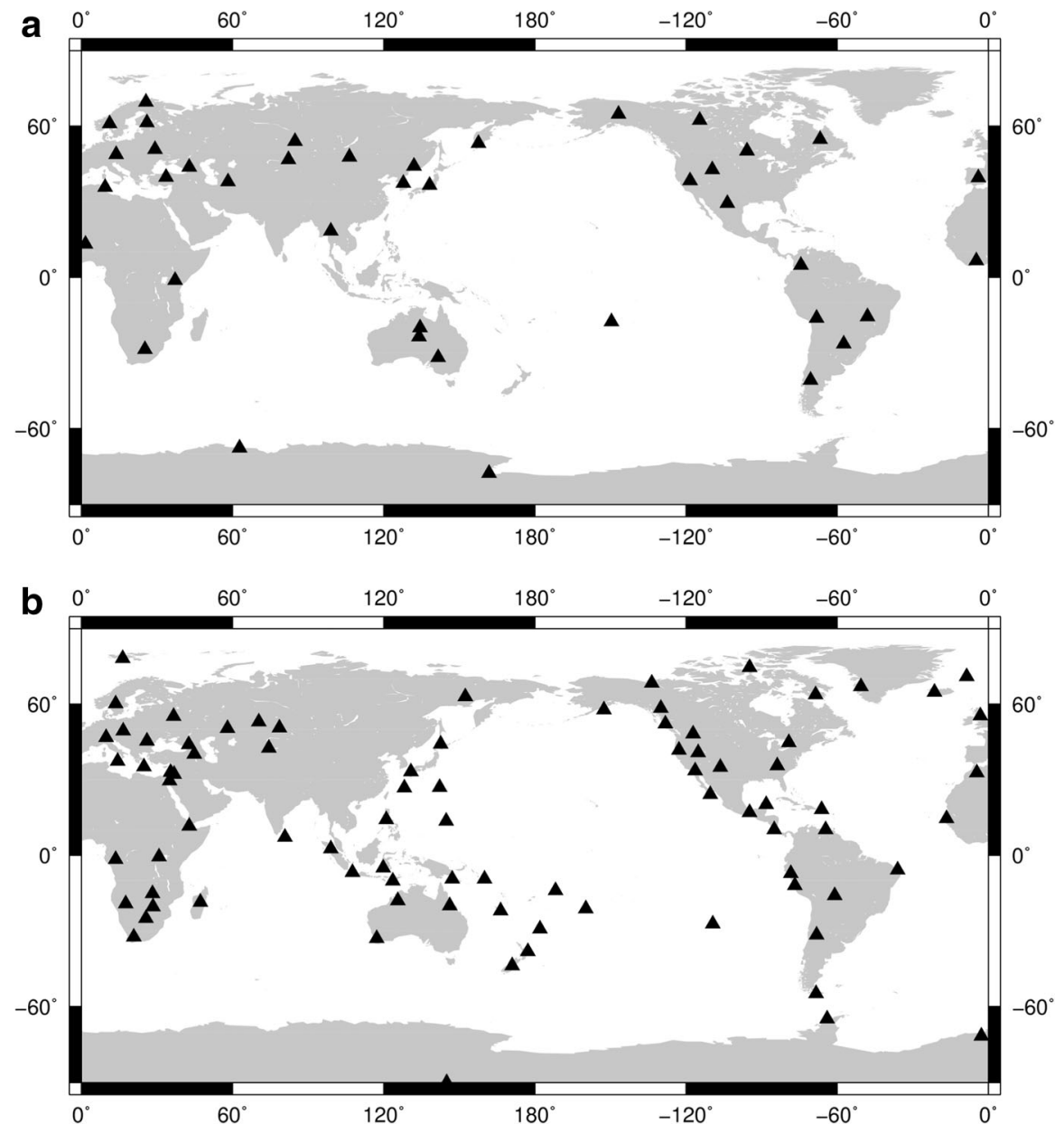

Figure 3

a Location of the primary seismic stations that contributed to the present analysis. b Location of the auxiliary seismic stations that contributed to the present analysis. c Location of the primary infrasound stations that contributed to the present analysis. d Location of the primary hydroacoustic stations that contributed to the present analysis. Note that no T-stations were used in this component of the present analysis

and, in the case of short-period seismic sensors, only spectral data at frequencies higher than $0.1 \mathrm{~Hz}$ were allowed to contribute to the analysis.

Event bulletins can be used as an additional measure to ensure that correctly calibrated data are contributing to the seismic low and high noise models. Event $\mathrm{mb}$ and Ms magnitude residuals (i.e., network magnitude estimate minus the station magnitude estimate) were plotted as a function of time for each station for the time duration under consideration and a station allowed to contribute to the global noise models if the Ms and mb magnitude residuals were less than 0.3 magnitude units. An example of a station with data that is correctly calibrated and unlikely to bias the global noise models is shown in Fig. 5. An example of a station with data that may have a calibration error and could bias the global noise models if used is shown in Fig. 6.

The IDC global seismic low and high noise curves for 2010, referred to here as IDC2010_LS and IDC2010_HS, respectively, are shown in Fig. 7. Also shown for comparison are the NLNM and NHNM curves from PETERSON (1993). The IDC2010_LS and NLNM curves are in good agreement, particularly in the low noise case. The microseism peak is slightly lower in the case of the IDC2010_LS model; station AAK (Ala Archa, Kyrgyzstan) was found to be 

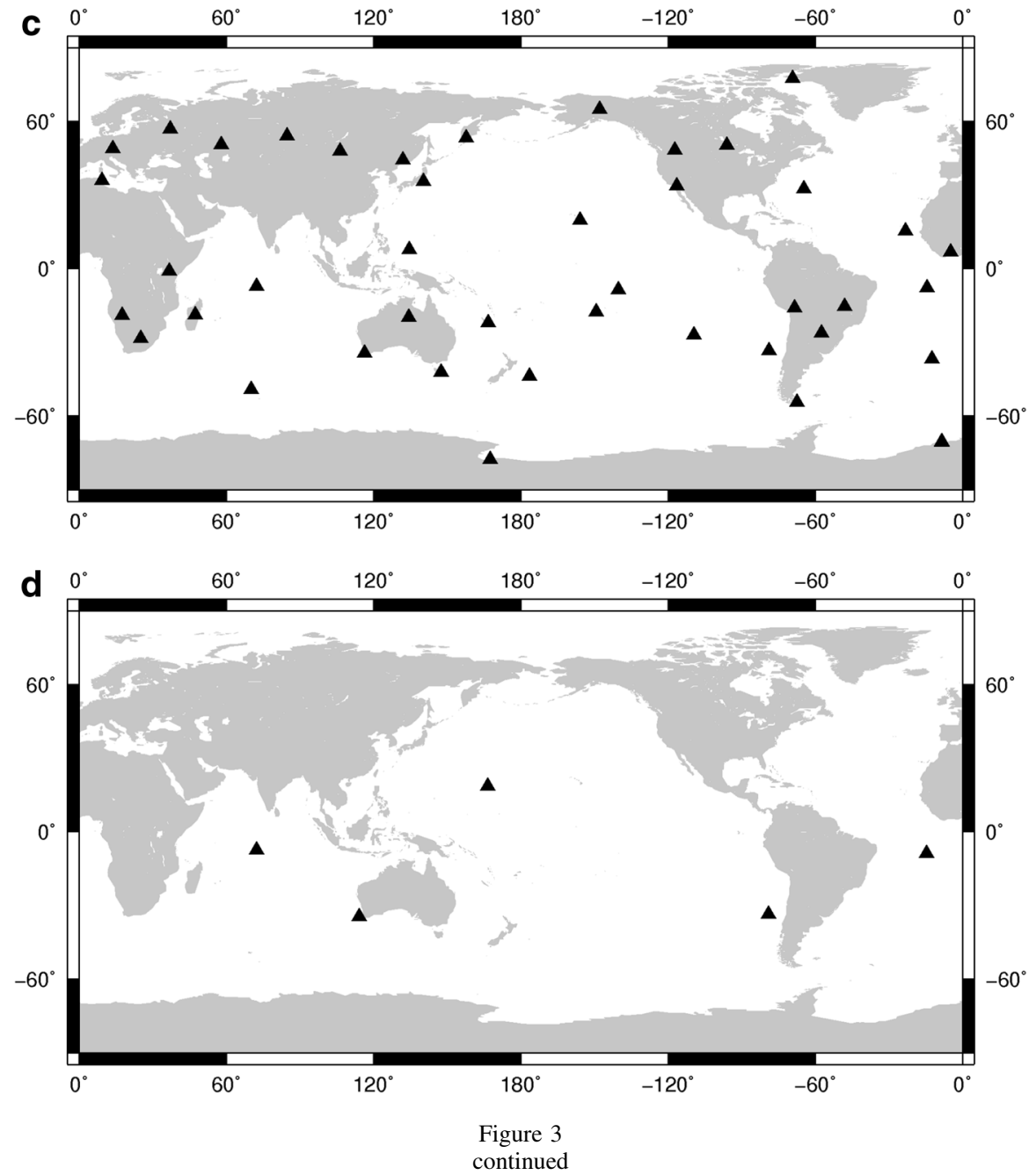

largely responsible for this slight deviation. In order to better resolve the contribution of this station to the low noise curve, data for the entire year, i.e., every hour in the day, for station AAK was processed.

Fairly good agreement exists between IDC2010_HS and the NHNM. The microseismic peak is slightly elevated in the IDC2010_HS model compared to the NHNM model, which is found to be due largely to station BORG (Borgarfjordur, Asbjarnarstadir, Iceland). This station commenced operation in 1994, so it would not have contributed to the PETERSON (1993) analysis. Waveform data for stations BORG was computed each hour for the entire year to better resolve its contribution to the IDC2010_HS model.

\subsection{Hydroacoustic}

Once again a random time shift of up to $6 \mathrm{~h}$ is applied to the requested processing time in order to reduce contamination from regular cultural noise.

The IDC global hydroacoustic low and high noise curves for 2010, referred to here as IDC2010_LH and IDC2010_HH, respectively, are shown in Fig. 8. Stations that contributed to these curves are shown in Fig. 3d. No T-stations were used during this analysis, so only in-water hydroacoustic signals contributed to the global noise curves. The low and high noise curves differ by a constant $20 \mathrm{~dB}$ from $0.01 \mathrm{~Hz}$ to around $6 \mathrm{~Hz}$ where the low noise curve begins to register shipping noise, making the curve relatively 


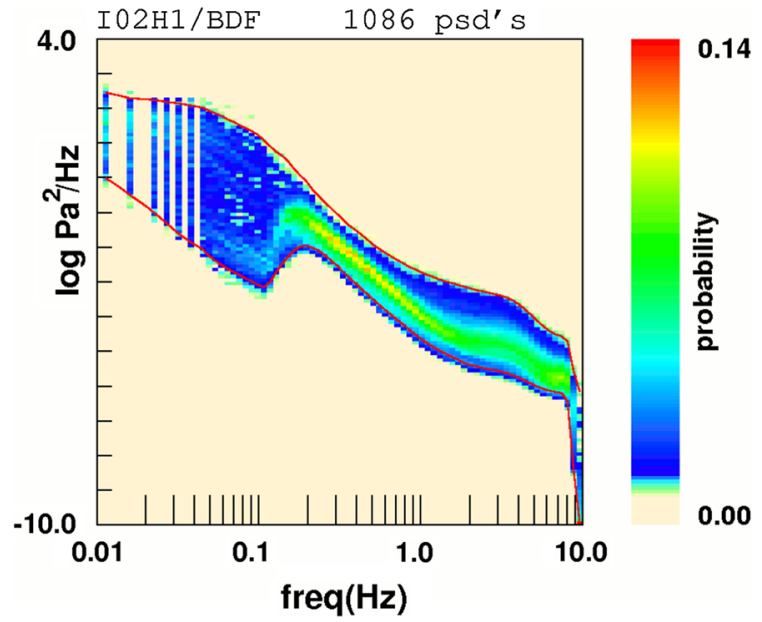

Figure 4

The PDF obtained for sensor I02AR/I02H1 for the year 2010. The low and high noise curves as shown in red are determined by plotting the PDF function at the 5 and $95 \%$ probability levels, respectively

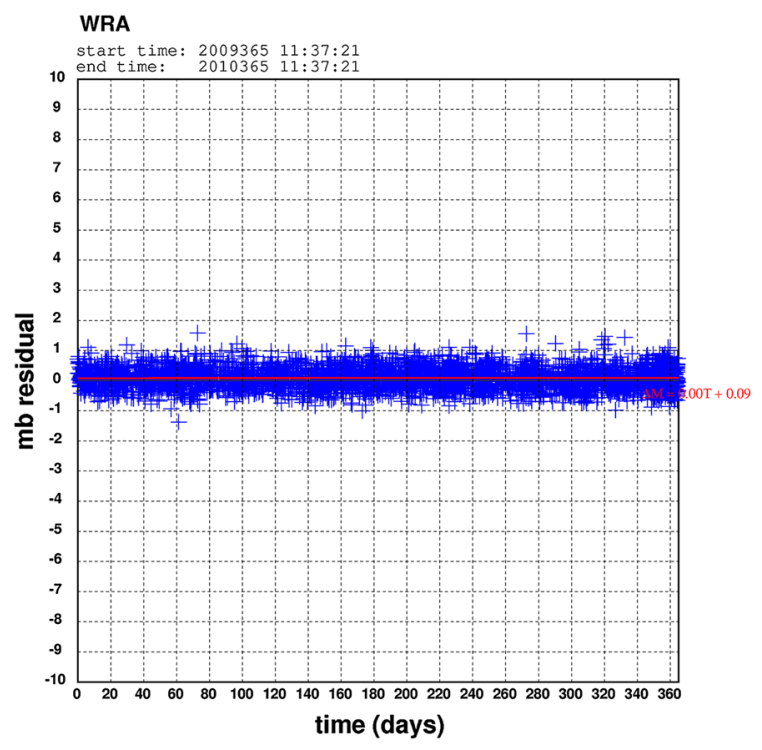

Figure 5

Event mb magnitude residual as a function of time for station WRA during 2010. No bias exists in the magnitude estimate suggesting the calibration for this station is correct

flat out to around $100 \mathrm{~Hz}$. The microseismic peak at around $5 \mathrm{~s}$ period is clearly visible in both curves. It is worth noting that the microseismic energy is being measured directly in the water, which means that it is a more local measurement of surface wave activity than for seismic stations that receive microseism

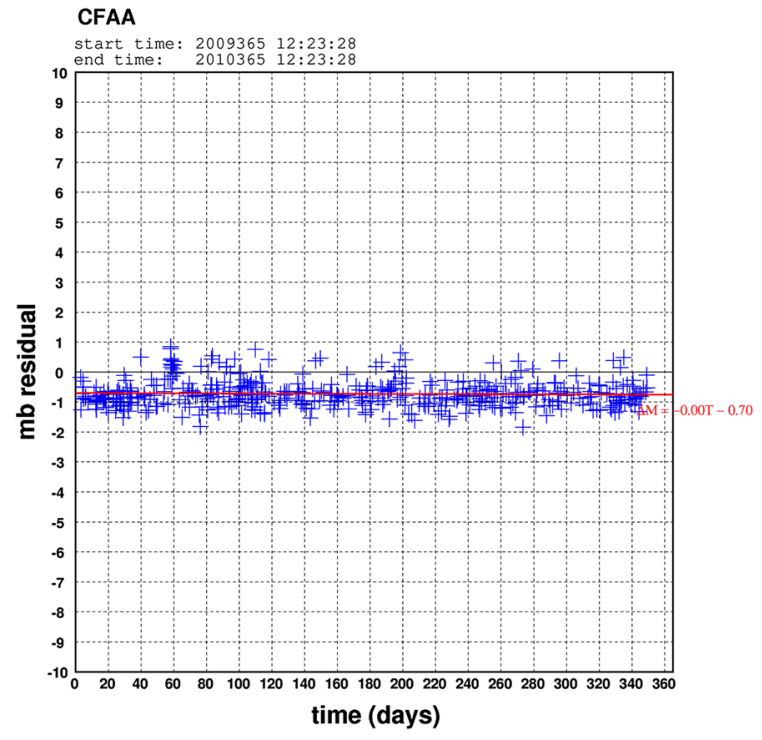

Figure 6

Event $\mathrm{mb}$ magnitude residual as a function of time for station CFAA during 2010. A clear bias of around -0.7 magnitude units exists for this station suggests a calibration error. The red line is the line of best fit through the data

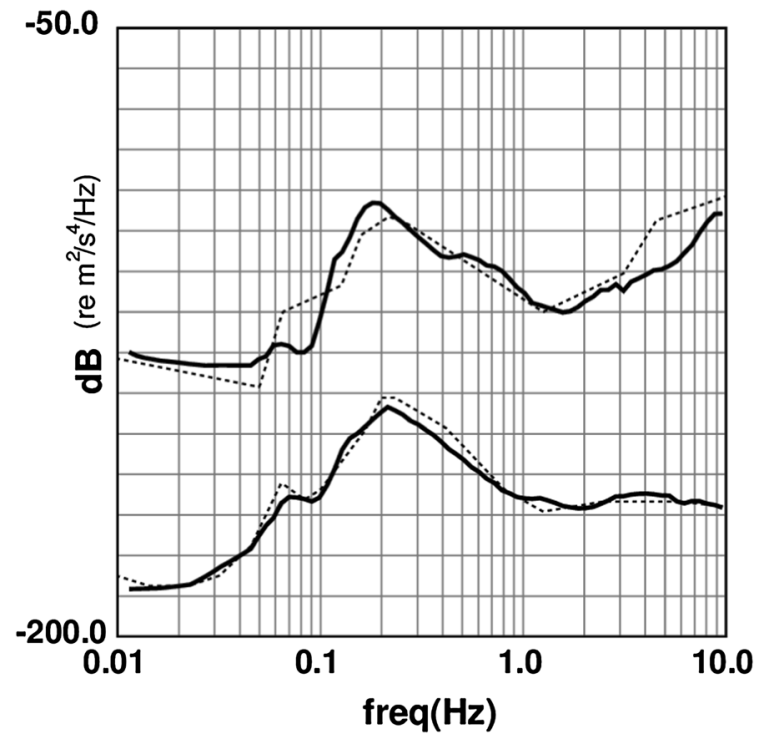

Figure 7

The global seismic low noise model IDC2010_LS and high noise model IDC2010_HS (solid lines) compared with the NHNM and NLNM of PETERSON (1993) (dashed lines)

energy from a large area of ocean after propagating through the crust. This explains why the low noise and high noise curves exhibit the same microseismic deviation. Also shown in Fig. 8 for comparison are the oceanic ambient noise curves presented in URICK 


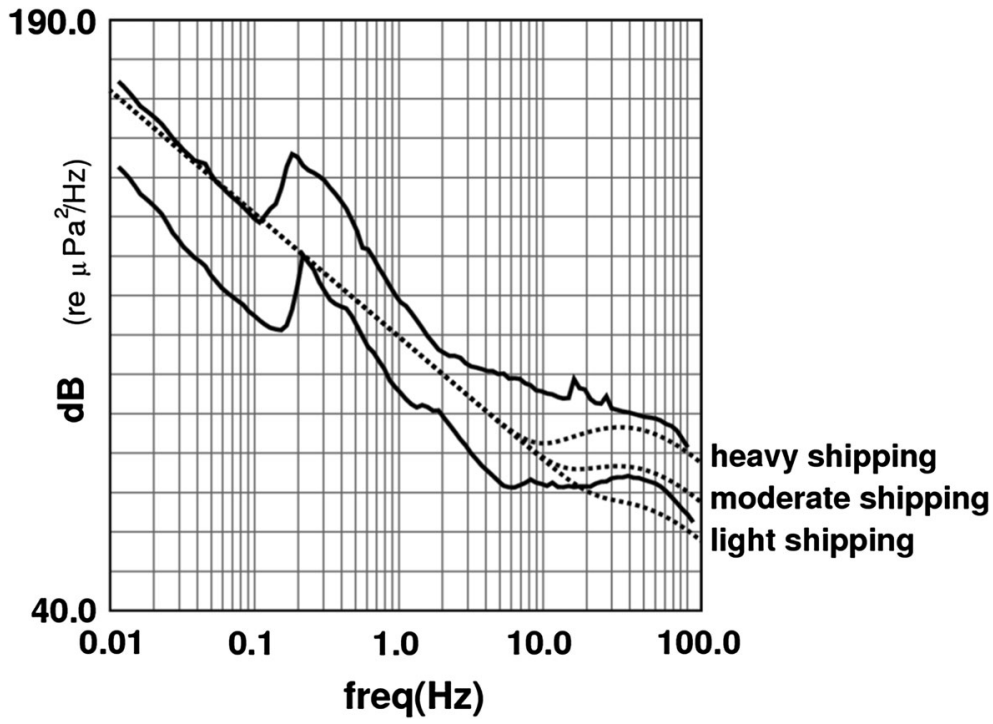

Figure 8

The global hydroacoustic low noise model IDC2010_LH and high noise model IDC2010_HH (solid curves) with Urick's noise curves superimposed (URICK 1984) for light, moderate and heavy shipping (dashed curves). Note that the wind component in the Urick curves was assumed to be zero, as the contribution due to the wind is not particularly significant below $100 \mathrm{~Hz}$

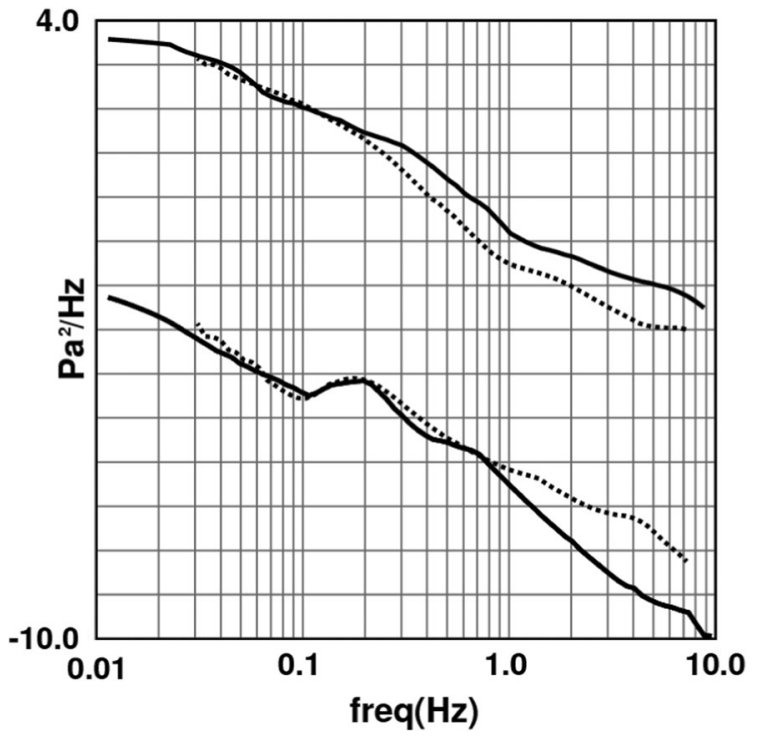

Figure 9

The global infrasonic low noise model IDC2010_LI and high noise model IDC2010_HI (solid lines) compared with a two times scaled version of the Bowman (2009) that can be assumed to be representative of the Bowman $(2005,2007)$ values (dashed lines)

(1984) for light to heavy shipping. Good quantitative agreement is observed to exist between these two sets of curves. The features in the high noise curve at

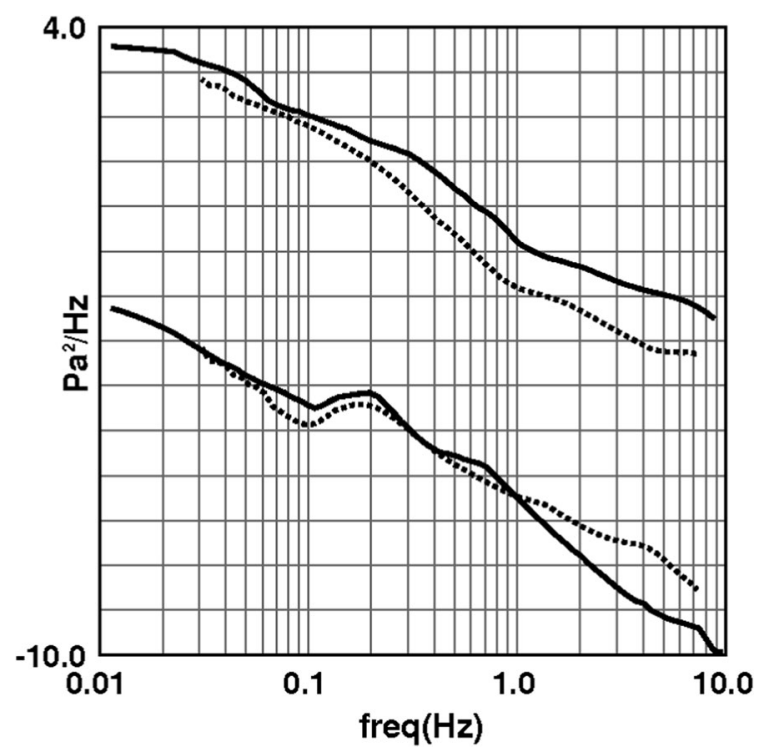

Figure 10

The global infrasonic low noise model IDC2010_LI and high noise model IDC2010_HI (solid lines) compared with those of BowmAN (2009) (dashed lines)

around 15 and $25 \mathrm{~Hz}$ are likely to be due to blue and fin whale calling (see, e.g., McCAuley et al., 2001; RICHARDSON et al. 1995). 

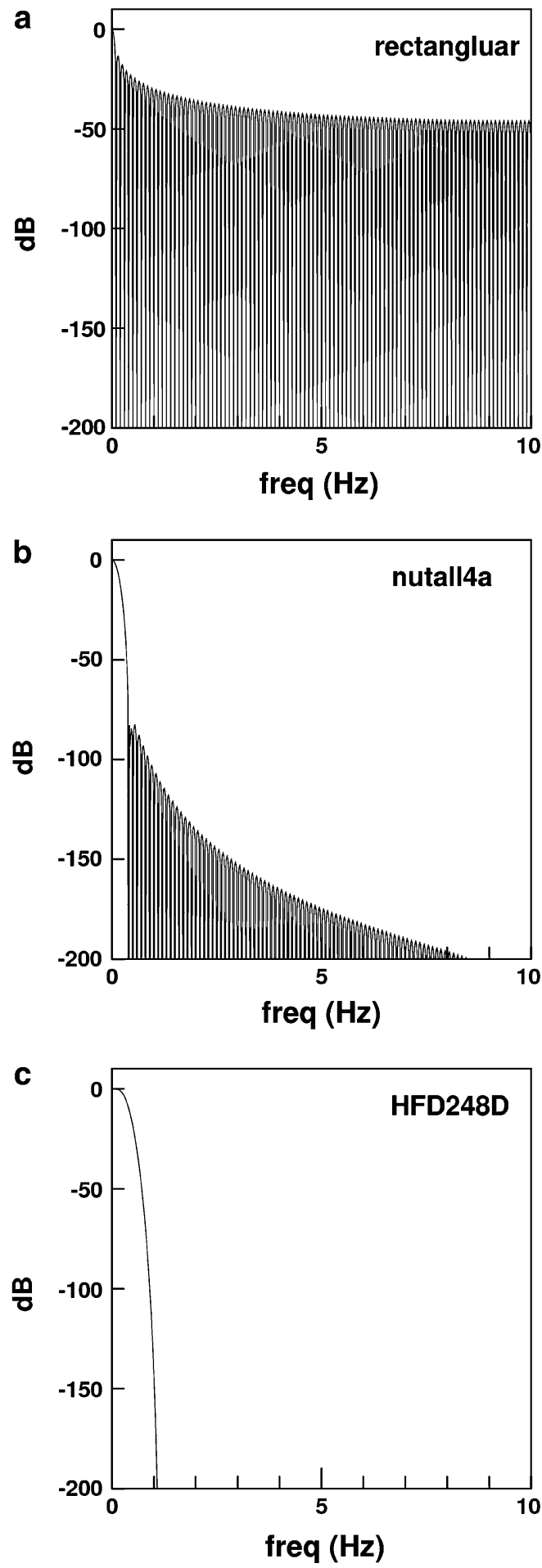

Figure 11

Transform of three spectral windows: a rectangular; b nutall4a; c HFD248D. See HeinZel et al. (2002) for a discussion on these last two windows

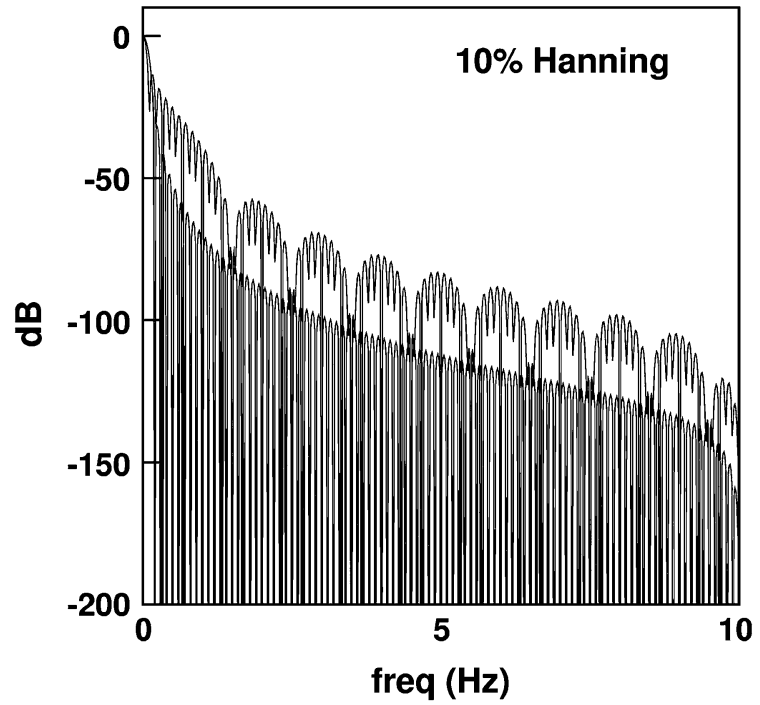

Figure 12

Transform of window function with a Hanning taper applied to the outer $10 \%$ of the window and with the inner $80 \%$ of the window function set to unity (upper curve). The transform of the usual Hanning window is also shown for comparison (lower curve)

\subsection{Infrasonic}

Infrasound data is requested at the local time: 03:30-04:30, 09:30-10:30, 15:30-16:30 and 21:3022:30 at each recording station in order to sample the coldest part of the night ( $\sim 4 \mathrm{am})$ and warmest part of the day $(\sim 4 \mathrm{pm})$, although it is appreciated these times may become a fairly inaccurate approximation for stations with extreme latitudes.

During the formulation of these noise curves station IS23, located at Kerguelen, was dropped from the high noise analysis as strong resonance peaks generated by the spatial filters significantly elevated the Power Spectral Densities at high frequencies, and would lead to a bias in the global high noise model.

The IDC global infrasonic low and high noise curves for 2010, referred to here as IDC2010_LI and IDC2010_HI, respectively, are shown in Fig. 9. Also shown in Fig. 9 are the global low and high infrasonic noise curves reported by Bowman et al. (2009) that have been scaled by two in the PSD's and can be assumed to be representative of the 2005/2007 results, since Bowman et al. (2009) state their 2009 results differ from the earlier 2005/2007 results by a factor of two, the latter results being half of the 
earlier results. The revised noise curves presented in Bowman et al. (2009) are shown in Fig. 10. Inspection shows that the Bowman $(2005,2007)$ low noise curves and IDC2010_LI curve are in close agreement around the microbarom peak. The IDC2010_LI curve drops below the Bowman curve at higher frequencies. This is due to the inclusion of station IS55 in the IDC2010_LI curve where it was excluded from the Bowman analysis. Station IS55, located at Windless Bight in the Antarctic, employs sensors manufactured by Chaparral Physics that are known to have very low self-noise at high frequencies. This station was excluded in the Bowman analysis because the authors felt that snow covering the spatial filters may artificially lower the recorded noise levels. However, it was included in this analysis as it is thought that the Chaparral sensors make an important contribution to the high frequency end of the noise curves that would otherwise be ignored. It is felt here that the curve obtained with the IS55 sensors included would more likely be closer to reality than those obtained leaving them out. The revised noise curves presented in Bowman et al. (2009) shown in Fig. 10 are consistently out in the value of the PSD's by a factor of 2, suggesting from this analysis that the original analysis of BowmAN et al. $(2005,2007)$ is correct and a systematic error was introduced in the more recent work.

\section{Conclusions}

Data recorded in 2010 from the CTBTO IMS seismic, hydroacoustic and infrasound networks has been used to infer global low and high ambient noise curves.

The determined seismic global low and high noise curves are found to be in excellent agreement with those established by Peterson (1993), whereas the global infrasound low and high noise curves are found to be in general agreement with the Bowman (2005, 2007) models, but disagree with the revised models presented in Bowman (2009), which seem to have a factor of 2 error in the determination of the PSD. The hydroacoustic global low and high noise curves both exhibit contributions from the microseisms at low frequencies and shipping noise at high frequencies. Whale noise is a feature of the high noise curve at 15 and $25 \mathrm{~Hz}$.

\section{Acknowledgments}

The authors would like to thank Dr. Roger Bowman for providing copies of his infrasound global noise models.

Disclaimer The views expressed in this paper are those of the authors and do not necessarily reflect those of the Preparatory Commission.

Open Access This article is distributed under the terms of the Creative Commons Attribution License which permits any use, distribution, and reproduction in any medium, provided the original author(s) and the source are credited.

\section{Appendix: Estimation of the Power Spectral Density}

The selection of a finite-time interval of digitally sampled data, with supposedly constant sample rate, is equivalent to taking the analogue signal and applying two processes:

1. Sampling using the periodic Dirac Comb function $D(t)=\sum_{k=-\infty}^{\infty} \delta(t-k T)$, where $T$ is the sampling period

2. Windowing using the box-car window function $w(t)=\left\{\begin{array}{cc}1 & \text { when } 0 \leq t \leq K T \\ 0 & \text { otherwise }\end{array}\right.$, where it is assumed that time zero is at the beginning of the box-car function, which is of duration $K T$.

The Fourier transform now becomes:

$$
F(f(t) D(t) w(t)) \equiv F(\omega) * D(\omega) * W(\omega)
$$

where asterisk $(*)$ indicates the convolution function, and the capitals indicate Fourier Transformation, and $D(\omega)=\sum_{k=-\infty}^{\infty} \delta\left(\omega-k \frac{2 \pi}{T}\right)=\sum_{k=-\infty}^{\infty} \delta\left(\omega-\omega_{k}\right)$. The Fourier transform of the window function is seen to control the measured frequency content. Examples for several spectral windows are shown in Fig. 11. The phenomenon of 'spectral leakage' is clearly visible in this figure, where energy due to the nonperiodic nature of the windowing process is migrated to higher-frequencies. In order to reduce the contri- 
bution of spectral leakage to the measured frequency content, it is common practice to taper the window function $w$, such that the side-lobes are smaller, invariably at the expense of losing some frequency resolution, but generally at the gain in amplitude resolution. Careful comparison of the properties of a large number of window functions is given in HeINZEL et al. (2002), where the different windows are classified according to their ability to either resolve frequencies, as in the box-car window (Fig. 11a), resolve amplitudes, such as the flat-top windows (Fig. 11c), or be general purpose with some capability in both areas, such as the nutall windows (Fig. 11b). In this work we use the nutall4a window function of HeINZEL et al. (2002) whose transform is displayed in Fig. 11b. This is a general-purpose window that has good amplitude and frequency resolution with side lobes that are around $100 \mathrm{~dB}$ below the main lobe. Note that it has become common practice in the literature to apply a fractionally tapered window to waveform data, so that, for example, only the first and last $10 \%$ of the window function differs from unity. This can lead to significantly degraded behaviour and should be avoided. Figure 12 shows the window transform function for a window function that consists of a hanning taper applied to the first and last $10 \%$ of the data.

Several additional concepts are important when considering the use of window functions as applied to the determination of Power Spectral Densities. The first of these is the notion of Incoherent Power Gain. The spatially-extended nature of the main lobe of the window transform function, generally extending across several frequency bins, allows the window to gather energy from those neighbouring bins. HARRIS (1978) shows that if $N_{0}$ is the noise-power per bin, then the total power $P_{\mathrm{w}}$ collected by the window function is $P_{W}=\frac{N_{0}}{2 \pi} \int_{-\pi / \Delta}^{\pi / \Delta}|W(\omega)|^{2} d \omega=\frac{N_{0}}{\Delta} \sum_{j=0}^{n-1} w_{j}^{2} \equiv$ $\frac{N_{0}}{\Delta} I_{W}$-where $n$ is the number of samples, $\Delta$ is the sample rate, and $I_{W}$, which just is the sum of the squares of the window coefficients, is defined to be the Incoherent Power Gain of the window. We are therefore in a position to write an expression for the PSD of the finitelength digitally-sampled analogue signal $f(t)$. It is: $P_{\mathrm{SD}}\left(\omega_{j}\right)=\frac{2\left|F\left(\omega_{j}\right)\right|^{2}}{\Delta I_{W}}$ where $\omega_{j}=\frac{2 \pi j}{n \Delta}$ for $j=0, \ldots$, $n / 2$, is the jth frequency picket, and $F$ is the output of a unitary Fourier Transform algorithm. Here, we are taking into account the contribution of the negative frequencies with the factor 2 . It is important to note that $P$ is the power spectrum contained in each frequency bin, i.e., PSD, and not the power spectrum of an individual spectral component. To determine the power contained in an individual line spectra the concept of the Coherent Power Gain is useful. Application of Eq. (1) to the elemental waveform $f(t)=A e^{i \omega_{k} t}$ yields the result.

$$
F(f(t) D(t) w(t))=A \sum_{j=-\infty}^{\infty} w_{j} \delta\left(\omega-\omega_{k}\right) * \delta(\omega-
$$
$\left.\omega_{j}\right)=A \sum_{j=-\infty}^{\infty} w_{j}$, where $w_{j}$, are the values of the window function at times: $t+j T$ for $j=-\infty, \ldots, \infty$. In such a case $_{p_{2}}$ the Power Spectrum is given by $P_{S}\left(\omega_{j}\right)=\frac{2\left|F\left(\omega_{j}\right)\right|^{2}}{C_{W}}$, where $C_{W}$, which is the square of the sum of the window coefficients, is the Coherent Power Gain of the window. One can then estimate the amplitude of the line spectra by taking the square root of $P_{S}\left(\omega_{j}\right)$. The quantity $\beta$ is known as the Equivalent Noise Bandwidth of the Window function and through straightforward multiplication allows one to convert $P S D$ to $P S$ and vice versa.

The Recommended Overlap Value (ROV) for the nutall4a window is $68 \%$ (HeINZel et al., 2002), implying a total of 63 three-min segments to be evaluated.

\section{REFERENCES}

Bowman, J.R., Baker, G.E., and Bahavar, M, 2005. Ambient infrasound noise. Geophys Res. Lett. 32. L09803, doi:10.1029/ 2005 GL022486.

Bowman, J.R., ShIELds, G., O'Brien, M.S. 2007. Infrasound Station Ambient Noise Estimates and Models 2003-2006 Infrasound Technology Workshop, Tokyo, 13-16, November 2007.

Bowman, J.R., ShIELDS, G., O'Brien, M.S. 2009. Infrasound station ambient noise estimates and models 2003-2006 (Erratum), Infrasound Technology Workshop, Brasilia, Brazil, November 2-6, 2009.

HARRIS, F.J.,1978. On the use of Windows for Harmonic Analysis with the Discrete Fourier Transform. Proceedings of the IEEE 66 (1): 51-83. doi:10.1109/PROC.1978.10837.

Heinzel G., Rudiger, R., and Schilling R. 2002. Spectrum and spectral density estimation by the Discrete Fourier Transform (DFT), including a comprehensive list of window functions and some new flat-top windows. http://www.rssd.esa.int/SP/LISA PATHFINDER/docs/Data_Analysis/GH_FFT.pdf.

Lyons, R.G., 1997. "Understanding Digital Signal Processing", Addison-Wesley. 
MATLAB, 2008. MATLAB version 7.7.0. Natick, Massachusetts: The MathWorks Inc.

McNamara, D.E. and R.P. Buland, 2004. Ambient Noise Levels in the Continental United States, Bull. Seism. Soc. Am., 94, 4, $1517-1527$.

McCauley, R.D., Jenner C., Bannister J.L., Burton C.L.K, Cato, D.H., and Duncan., A. 2001. Blue whale calling in the Rottnest trench-2000, Western Australia. Prepared for Environment Australia, from Centre for Marine Science and Technology, Curtin University, R2001-6, 55 pp.

Peterson, J., 1993. Observation and modeling of seismic background noise, U.S. Geol. Surv. Tech. Rept., 93-322, 1-95.
Richardson, W.J., Greene, C.R., Malme, C.I, and Thomson, D.H. 1995. Marine Mamals and Noise, Academic Press, San Diego. pp 576. SCHREIER, P.J, and ScharF, L.L, 2010. Statistical signal processing of complex-valued data: the theory of improper and noncircular signals. Cambridge University Press. pp. 330.

URICK, R.J. 1984. Ambient noise in the sea. Report No. 20070117128. Undersea Warefare Technology Office, Naval Sea Systems Command, Department Of The Navy, Washington DC. Welch, PD, 1967. The Use of Fast Fourier Transform for the Estimation of Power Spectra: A Method Based on Time Averaging Over Short, Modified Periodograms, IEEE Transactions on Audio Electroacoustics, Volume AU-15 pp. 70-73.

(Received September 30, 2011, revised August 6, 2012, accepted August 12, 2012, Published online September 8, 2012) 